\title{
Research on the Method of Materiel Maintainability Qualitative Requirement Demonstration Based on Virtual Simulation
}

\author{
XU Da1 \\ Department of Arms Engineering, \\ Academy of Armored Force Engineering, \\ Beijing, China, \\ 283009953@qq.com; \\ WANG Bao-qi2 \\ Department of Arms Engineering, \\ Academy of Armored Force Engineering, \\ Beijing, China, \\ 7246141@qq.com
}

\author{
WU Xi3 \\ Department of Arms Engineering, \\ Academy of Armored Force Engineering, \\ Beijing, China, \\ 1113609446@qq.com
}

\begin{abstract}
Aiming at the disadvantage of existing materiel maintainability quantitative requirements demonstration method, this paper studied the project of the materiel maintainability qualitative requirement demonstration Based on virtual simulation, in order to determine the materiel maintainability qualitative requirement, the accessibility and visibility as an example to illustrate the virtual demonstration method. It is significant for developing maintainability demonstration and increasing materiel maintainability in the course of materiel digital mock-up designing.
\end{abstract}

Keywords- maintainability; qualitative requirement; virtual simulation

\section{INTRODUCTION}

Maintainability refers to the ability that time under specified conditions and provisions, according to prescribed procedures and methods for maintenance, to maintain or restore its provisions state[1].Maintainability qualitative requirement reflects impossible or difficult to quantitatively describe the maintainability requirement, based on ensuring that the materiel is convenient for maintenance of this basic point, from the different aspects of the proposed design stage should be to achieve the design requirement[2], thus realizing the materiel maintainability growth.

The traditional materiel maintainability qualitative requirement demonstration is mainly relying on physical prototypes. However, in general, the lack of a physical prototype at the materiel design stage, some important maintainability qualitative requirement demonstration work can't be carried out. This has seriously affected materiel maintenance design and materiel design parallel to carry out. Using virtual simulation technology in the materiel maintainability qualitative requirement demonstration method, it is an important practical significance for improve the materiel maintainability[4].

\section{DETERMINE THE MATERIEL MAINTAINABILITY QUALITATIVE REQUIREMENT}

Determine the materiel maintainability qualitative requirement is the foundation and prerequisite for the virtual demonstration of the materiel maintainability qualitative requirement. Maintainability demonstration is the purpose of the assessment of whether or not the materiel can meet the maintainability design requirement. According to GJB, maintainability design generally requirement including: simplicity, accessibility, visibility, standardization, human factors engineering requirements. The maintainability design criteria included content is more, is the product of the maintainability design generally guideline. According to the characteristics of configuration and maintenance actual situation, combined with the design standards, determine the applicability of materiel development of digital prototype of maintainability qualitative requirement. As shown in Figure 1.

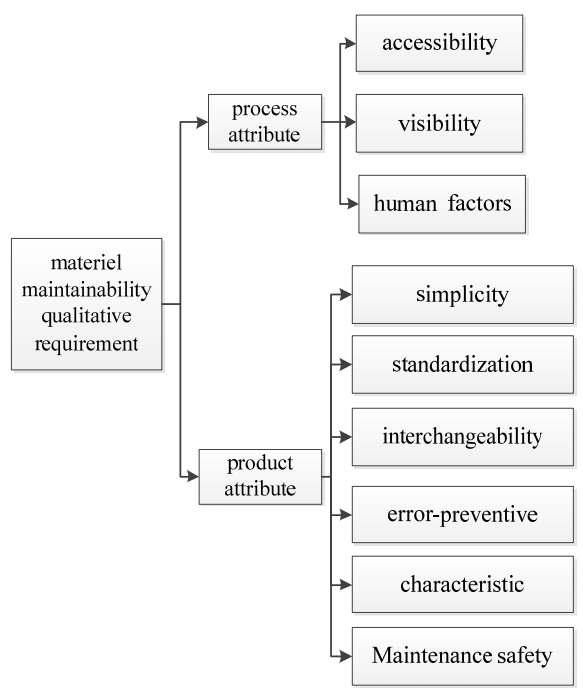

Figure 1. Materiel maintainability qualitative requirement.

The materiel maintainability qualitative requirement of the different characteristics depending on the demonstration method can be divided into two categories. First class attribute is called the process attribute. The qualitative requirement of this attribute maintenance needs through a combination of virtual maintenance process simulation technology in order to get a specific demonstration result, 
which mainly include accessibility, visibility and human factors engineering. Accessibility reflects the ease of maintenance personnel close to the faulty components during maintenance; Human factors engineering is the relationship of the human body and the operating environment in materiel maintenance process

The second class attribute is called the demonstration element of product attribute. Analysis for the product itself, the attributes of the maintainability factors don't requires a combination of virtual simulation technology in materiel maintenance, as long as the detailed CAD data model can be determined, the result is obtained. Including simplicity, standardization, interchangeability, error prevention ability and maintenance of security requirement.

\section{THE OVERALL PROGRAM OF MAINTAINABILITY VIRTUAL DEMONSTRATION}

By selecting Virtual maintenance human model, call maintenance action model library of database and maintenance tools, to complete virtual maintenance dynamic simulation of the digital prototyping CAD data model, Aim to the different characteristics of the digital prototype maintainability qualitative requirement at materiel development stage. Using different demonstration methods to verify the maintainability qualitative requirement. The overall process is shown in Figure 2.

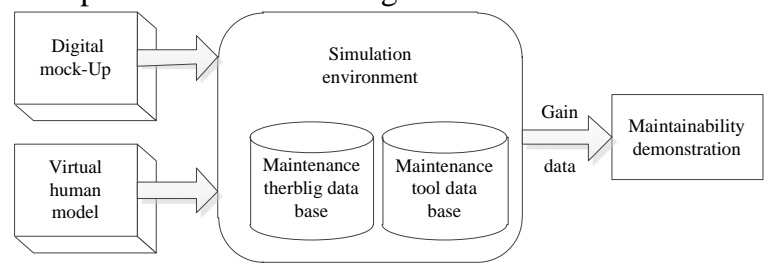

Figure 2. Flowchart of evaluation of maintainability.

\section{A. The method of accessibility demonstration}

Accessibility reflects that the maintenance working space, maintenance access can have an effect on maintenance work. Analysis of maintenance personnel have access to the pending maintenance components to determine whether to be reach components within the scope of upper limb up to the maintenance personnel.

In materiel simulation disassembly process, attention to the selection of suitable percentile human model. This will ensure that the simulation result reflect the actual maintenance situation of most of the maintenance personnel. The maintenance personnel with bare hands or maintenance tools direct access to the operating area, in order to achieve the operation of various types fasteners. Determine the maintenance personnel in the arm throughout the disassembly process can reach the boundary of the coverage of the envelope ball. In the envelope ball is the arm of maintenance personnel can be reached to the operating position. However, outside of the envelope ball is the arm of maintenance personnel can't be reached to the operating position, as shown in Figure 3.

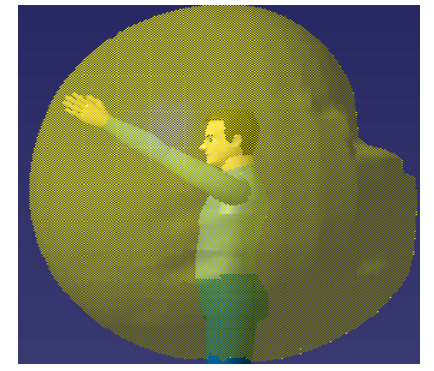

Figure 3. Reach zones of the arm.

By analyzing the dynamic simulation results, when the pending maintenance components in the envelope ball, a score of 1 ; otherwise score 0 . In the presence of channel need to be analyzed that whether the maintenance personnel can smooth through the maintenance channel for maintenance , as with other components or channel interference can be applied to the collision detection algorithms[3]to judge the virtual human in the process of maintenance, whether can smoothly through the channel. When the maintenance personnel can smooth through the maintenance channel for maintenance, a score of 1 ; otherwise score 0 , the flowchart of accessibility demonstration, as show in Figure 4.

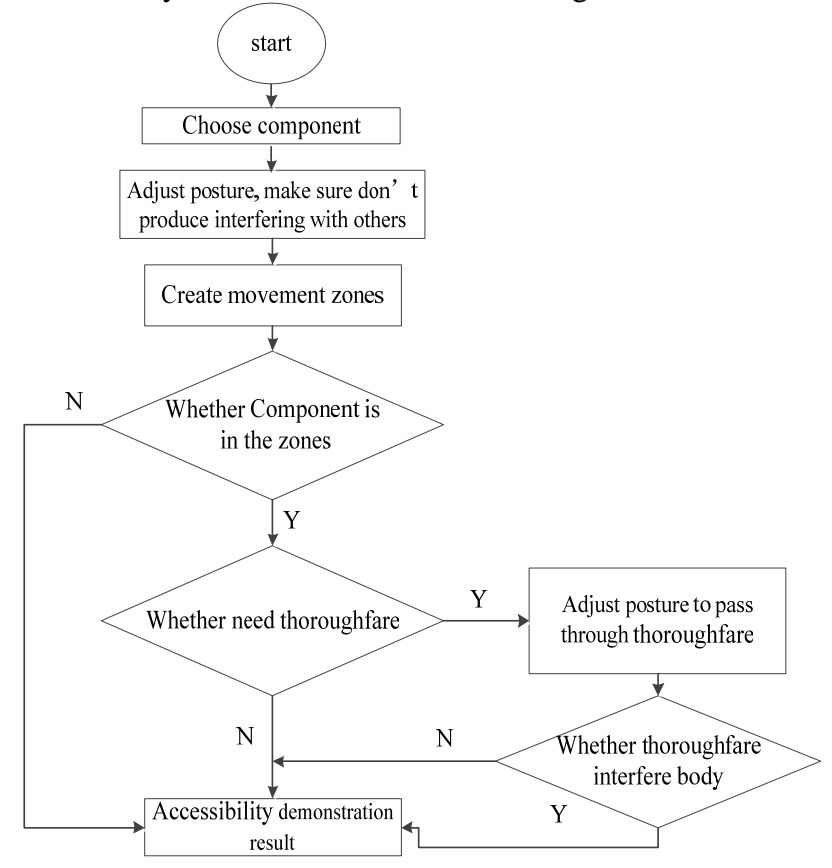

Figure 4. Flowchart of accessibility demonstration.

\section{B. The method of visibility demonstration}

GJB respectively on the vertical and horizontal visual fields make the relevant provisions. The visual fields range of the human eyes is all the space of to be perceived in the horizontal and vertical plane when the head is maintained in a fixed position and the eyes move in order to monitor the target. The horizontal visual fields range of the human horizontal centerline around the $15^{\circ}$ is the human optimal level of visual fields, the centerline around $35^{\circ}$ is the human 
eyes in the horizontal direction maximum visual fields range .The normal line of the human eye vertical visual fields is $15^{\circ}$ below the horizontal line . The normal line of sight up and down $15^{\circ}$ is the optimal vertical visual fields, the maximum visual fields is above the normal line of sight $40^{\circ}$ and $20^{\circ}$ below.

The maintainability virtual demonstration method is to restore the true visual fields range of the human eyes. To demonstration the maintenance components visibility from the perspective of 3D, whether or not qualified. From physiology angle analysis. The visual fields areas of the human eyes is the human eye as the center, the enveloping space curved surface of angled outward diffusion. The surface is an oval cross-section, is an elliptical cone that is the human eye vision cone analysis from the space angle. For the convenience of calculation, can be placed in the intersection point of the two pitch and sagittal .as show in Figure 5.

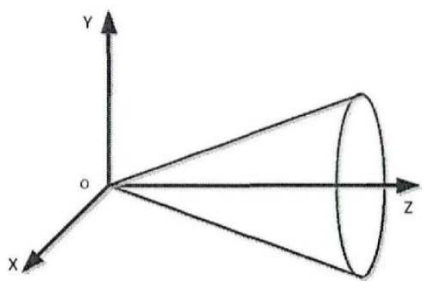

Figure 5. Vision cone of human.

Set the intersection point of the two pitch and sagittal as the coordinate origin $\mathrm{O}$. Use of the spatial solid geometric method to build a mathematical model of the vision cone[5],The optimal visual fields range and the maximum visual fields range of the following formula:

$$
\left\{\begin{array}{l}
\frac{x^{2}}{\left(\tan 15^{\circ}\right)^{2}}+\frac{y^{2}}{\left(\tan 15^{\circ}\right)^{2}}=z^{2} \\
\frac{x^{2}}{\left(\tan 35^{\circ}\right)^{2}}+\frac{y^{2}}{\left(\tan 35^{\circ}\right)^{2}}=z^{2}
\end{array}\right.
$$

For a point on an object $A$, The space coordinates is $A\left(x_{a}, y_{a}, z_{a}\right)$,identification of the point coordinates if meet: $\frac{x^{2}}{\left(\tan 15^{\circ}\right)^{2}}+\frac{y^{2}}{\left(\tan 15^{\circ}\right)^{2}} \leq z_{\alpha}^{2}$, it belong to the optimal visual fields range; if the coordinates meet: $\frac{x_{\alpha}^{2}}{\left(\cot 15^{\circ}\right)^{2}}+\frac{y_{\alpha}^{2}}{\left(\cot 15^{\circ}\right)^{2}}<z_{\alpha}^{2} \leq \frac{x^{2}}{\left(\tan 35^{\circ}\right)^{2}}+\frac{y^{2}}{\left(\tan 30^{\circ}\right)^{2}}$, it belong to the maximum visual fields range; if the coordinates meet: $\frac{x^{2}}{\left(\tan 35^{\circ}\right)^{2}}+\frac{y^{2}}{\left(\tan 30^{\circ}\right)^{2}} \leq z_{\alpha}^{2}$, it beyond the visual range.

According to the above mathematical model, combined with simulation visual cone, by judging the $3 \mathrm{D}$ space coordinates of the materiel maintenance components, the visibility of the materiel maintenance components can be verified, finally, give the final visibility evaluation result by the criterion of visibility evaluation.

\section{CONCLUSION}

This article from the maintainability design criteria to determine the maintainability qualitative requirement for materiel. By building virtual maintenance simulation process, given the method of the accessibility and visibility virtual demonstration, can overcome the lack of the materiel maintainability demonstration must rely on physical prototypes, long cycle, high cost .The research results show that the method has strong operability, on the future development of the maintainability qualitative requirement demonstration has an important reference value.

\section{REFERENCES}

[1] GAN Mao-zhi . Maintainability Design and Demonstration [M]. Beijing: National Defense Industrial Press, 1995.

[2] LV Chuan . Design , Analysis and Verification of Maintainability[M]. Beijing: National Defense Industrial Press, 2012.

[3] Liu Ying. Interactive reach planning for animated characters using hardware acceleration[D] . Pennsylvania : University of Pennsylvania, 2003.

[4] YAN Shi-chun, et al.. Summary of Collision Detection Algorithms in Virtual Maintenance Training System[J]. Machine Building \& Automation, 2012 (1) : 98-100.

[5] XIE Kai. Research on Maintainability Analysis and Evaluation Methods of High-speed Train Cab's Equipments[D]. Beijing Jiaotong University, 2011. 